\title{
Structure of conditioned sludge flocs
}

\author{
C.P. Chu ${ }^{\mathrm{a}}$, D.J. Lee ${ }^{\mathrm{a}, *}$, X.F. Peng ${ }^{\mathrm{b}}$ \\ a Department of Chemical Engineering, National Taiwan University, Taipei 10617, Taiwan \\ b Thermal Engineering Department, Tsinghua University, Beijing 100084, China
}

Received 3 December 2003; received in revised form 31 January 2004; accepted 4 February 2004

\begin{abstract}
Free settling tests, small-angle light scattering, microtome-slicing techniques, and confocal laser scanning microscopy were performed to examine how the cationic flocculation or freezing and thawing affected the floc structure. The floc size, internal pore size, mass fractal dimensions determined from free-settling test or small angle light scattering test, aeral porosity, boundary fractal dimension and Sierpinski carpet fractal dimension of pore boundary from 2D slices, and the volume porosity, compactness, and the pores' box-counting fractal dimension from 3D reconstructed image, were estimated and compared. Cationic flocculation would produce large flocs with internal pores of shape resembling a long "tube" with rough surface. Freezing and thawing would produce flocs with internal pores with lower aspect ratio and a smoother boundary.
\end{abstract}

(C) 2004 Elsevier Ltd. All rights reserved.

Keywords: Floc structure; Freezing; Flocculation; Image analysis

\section{Introduction}

Sludge flocs are highly porous fractal-like aggregates of many primary particles. Li and Ganczarczyk [1] sliced the activated sludge flocs to examine cross-sectional morphology, confirming their fractal-like interior structure. An important parameter that characterizes a fractal object is the fractal dimension, $D(-)$, which corresponds to the space-filling capacity of an object. Free-settling tests [2] and small-angle light scattering (SALS) experiments (Guan et al., 1998) were widely used to probe the fractal dimensions of the sludge flocs.

Conventional models assumed that molecular diffusion controlled the intrafloc transport processes [3]. The revised, fractal-like floc model has been used to explore its hydrodynamic behavior and the coagulation characteristics [4,5]. Aggregates occurring naturally may have multi-level structure [6-8]. The simplest scenario for an activated sludge floc is fractals in fractal. Wu et al. [9] utilized both free-settling test and SALS experiments for demonstrating the "two-level" structural character-

\footnotetext{
*Corresponding author. Fax: + 886-2-2362-3040.

E-mail address: djlee@ccms.ntu.edu.hw (D.J. Lee).
}

istic of the sludge flocs. Only recently did researchers started to explore the three-dimensional structure of sludge flocs [10-13].

Cationic polyelectrolyte flocculation and freezing and thawing are widely adopted for sludge conditioning. Polyelectrolyte flocculation leads to the increase in floc size and decrease in the bound water content [14]. Freezing and thawing could convert the loose floc structure into a compact form [15]. Related reviews demonstrated a significant role of floc structure on the sludge settling [16-18]. Meanwhile, the information on the structural changes for flocs subject to chemical or physical conditioning is still largely lacking. In this work, we probed in detail the structure of wasteactivated sludge flocs, and estimated the aeral and volume porosities, and other geometric parameters that characterized the internal structure.

\section{Experimental}

\subsection{Samples}

Waste-activated sludge was sampled from the wastewater treatment facility at the Neili Bread Plant, 


\begin{tabular}{|llll|}
\hline Nomenclature & $g$ & gravitational acceleration $\left(=9.8 \mathrm{~m} \mathrm{~s}^{-2}\right)$ \\
& & $I$ & intensity of scattered light (dimensionless) \\
$A_{\mathrm{p}}$ & pore area $\left(\mathrm{m}^{2}\right)$ & $l_{\text {equi }}$ & characteristic side length $(\mathrm{m})$ \\
$d_{\mathrm{p}}$ & floc size $(\mathrm{m})$ & & number of triangular patches covering the \\
$C$ & solid content $\left(\mathrm{kg} \mathrm{m}^{-3}\right)$ & & pores (dimensionless) \\
$D_{\mathrm{B}}$ & boundary fractal dimension (dimensionless) & $p_{\mathrm{p}}$ & pore perimeter $(\mathrm{m})$ \\
$D_{\mathrm{F}}$ & fractal dimension by free-settling test (dimen- & $Q$ & scattered wave vector $(\mathrm{m})$ \\
& sionless) & $u_{\mathrm{ST}}$ & free-settling velocity $\left(\mathrm{m} \mathrm{s}^{-1}\right)$ \\
$D_{\mathrm{P}, 3}$ & box-counting fractal dimension (dimension- & $V$ & pore volume $\left(\mathrm{m}^{3}\right)$ \\
& less) & $\mu$ & fluid viscosity (Pa s) \\
$D_{\mathrm{S}}$ & fractal dimension by light-scattering test & $\varepsilon_{2 \mathrm{D}}$ & two-dimensional porosity (dimensionless) \\
& (dimensionless) & $\varepsilon_{3 \mathrm{D}}$ & three-dimensional porosity (dimensionless) \\
$D_{\mathrm{SC}}$ & Sierpinski carpet fractal dimension (dimen- & $\phi$ & equipment constant (dimensionless) \\
& sionless) & &
\end{tabular}

Presidential Enterprise Co., Taoyuan, Taiwan. The total solid content (TS) was measured as $9350 \mathrm{mg}^{-1}$. The $\mathrm{pH}$ value of original sludge was around approximately 7.0. A particle sizer (LS230, Coulter, USA), based on laser diffraction technique, estimated the volume-average floc diameter of original sludge as $73.6 \mu \mathrm{m}$.

\subsection{Conditioning}

The polyelectrolyte flocculant, polymer T-3052, is a cationic polyacrylamide with an average molecular weight of $10^{7} \mathrm{~g} \mathrm{~mol}^{-1}$ and a charge density of $2.27 \mathrm{meq} / \mathrm{g}$. A sludge sample was flocculated in a mixing vessel at $200 \mathrm{rpm}$ for $5 \mathrm{~min}$ and then $50 \mathrm{rpm}$ for a further $20 \mathrm{~min}$. The flocculant dose was set to $40 \mathrm{~g} \mathrm{~kg}^{-1} \mathrm{DS}$, which caused charge-neutralization of the sludge flocs (zeta potential data not shown).

Sludge freezing was performed in glass containers with a radius of $2.3 \mathrm{~cm}$ and a height of $4.0 \mathrm{~cm}$, which were placed in a cool room at $-16.5^{\circ} \mathrm{C}$. The sludge sample was completely frozen in $2 \mathrm{~h}$ and then cured for a further $22 \mathrm{~h}$. The frozen sample was thawed at room temperature for another $6 \mathrm{~h}$.

\subsection{Evaluation of sludge dewaterability}

Capillary suction time (CST) tests were conducted using the CST equipment proposed by Lee and Hsu [19]. The CST value was then converted to the coefficient of filterability $\chi$, defined as follows [20]:

$\chi=\frac{\phi \mu C}{\mathrm{CST}}$,

where $C$ is the solids content of the sludge, $\mu$, the viscosity of the fluid, and $\phi$ is a dimensionless instrument constant (0.06 in our case).

Settling rate tests were performed in several 1-1 graduated cylinders. The sludge was allowed to settle and the height of sludge-liquid interface and the time were recorded.

\subsection{Free settling}

A glass tank $(10 \mathrm{~cm}(L) \times 10 \mathrm{~cm}(W) \times 30 \mathrm{~cm}(H))$ was used for floc-settling test. A floc was collected and released carefully using wide mount pipette from the top of the column. A CCD camera (MML2-120, Moritex, UK) with a microscopic lens (JAI $950 \frac{1}{3}$ in, Denmark) was used to record the settling process. The obtained image was transferred onto a personal computer using an interface card (Meteor PCI Bus, Matrox, Canada) and was processed using software INSPECTOR (Matrox, Canada). Balancing the gravitational force with the buoyancy force yields the following relationship [2]:

$u_{\mathrm{st}}=C_{1} \frac{g}{\mu} d_{\mathrm{f}}^{D_{\mathrm{F}-1}}$,

where $g$ is the gravitational acceleration, $C_{1}$ is a constant in the function that links floc permeability with effective density, and $D_{\mathrm{F}}$ is the fractal dimension determined by free settling [9]. The effects of non-spherical shape of the sludge flocs [17] were minimized by choosing nearly spherical flocs for measurement.

\subsection{Small-angle light scattering (SALS)}

Small-angle laser light scattering tests were performed using a particle sizer (Mastersizer 2000, Malvern, UK). When the aggregate exhibits fractal behavior, plotting the scattered intensity $I$ versus wave vector $Q$ on a $\log _{-}$ log plot yields a curve with a linear region, in the range $1 / R_{\mathrm{f}} \leqslant Q \leqslant 1 / R_{\mathrm{p}}$, where $R_{\mathrm{f}}$ and $R_{\mathrm{p}}$ are the radii of gyration of the scattering aggregates and the primary particles in the aggregates, respectively,

$1 \propto Q^{-D_{\mathrm{s}}}$

$D_{\mathrm{S}}$ represents the scattering fractal dimension [9]. 
This sizer also measured the median $\left(d_{\mathrm{f}}(0.5)\right)$ and volume-average floc size $\left(d_{\mathrm{f}}[4,3]\right.$, defined as $\left.\sum d_{\mathrm{f}, i} V_{i} / \sum V_{i}\right)$

\subsection{Microtome slicing}

The samples were first chemically fixed using a formalin buffer at $4^{\circ} \mathrm{C}$ for $24 \mathrm{~h}$ and embedded by agarose in the cassette. Dehydration was conducted by immersing the cake subsequently in ethanol/water solution of $50 \%, 70 \%, 90 \%, 95 \%$ and $100 \%(\mathrm{v} / \mathrm{v})$, respectively. The ethanol was then replaced by xylene/ ethanol solutions of $50 \%, 70 \%, 90 \%, 95 \%$ and $100 \%$ $(\mathrm{v} / \mathrm{v})$. The cake saturated with xylene was immersed in molten paraffin at $65^{\circ} \mathrm{C}$ overnight. Finally, the paraffinembedded cakes were cooled to $25^{\circ} \mathrm{C}$ in peel-off molds and solidified to form blocks for slicing [21]. The block was then sliced into sections of thickness $5 \mu \mathrm{m}$ using a microtome (Leitz Model 1400, Germany). The thin paraffin section was floated on a water bath and then transferred onto a glass slide. The slide was dried in air. Then, the slice was heated in an oven at $70^{\circ} \mathrm{C}$ for $10 \mathrm{~min}$ to melt the paraffin, before being dewaxed using xylene. Finally, the slice was stained using hematoxylin and eosin (H\&E) [22]. A phase-contrast microscope (LEICA DME, Germany) and a digital camera (NIKON COOLPIX 995, Japan) recorded the image of slices at a constant luminescence light (4000 LUX).

\subsection{Confocal laser scanning microscopy (CLSM)}

A confocal laser scanning microscope (CLSM; Leica TCS SP2, Germany) was used to observe the internal floc structure. This microscope was equipped with image processing software and an argon laser source was used to stimulate fluorescence. The sludge floc was imaged using $20 \times, 40 \times$, or $60 \times$ objectives, depending on the required resolution. The microscope scanned the samples at a fixed depth and the obtained image was digitized. Sludge samples were first chemically fixed and then embedded in agarose to perform fluorescence in situ hybridization (FISH). This study used DNA probes Eub 338 (labeled by rhodamine) to detect most eubacteria in biosolids. Its nucleotide sequence is available in Sørensen et al. [23]. After the probe was added, hybridization was performed at $50^{\circ} \mathrm{C}$ for $1 \mathrm{~h}$. The stained samples were washed three times at the same condition using hybridization buffer solution $(40 \%$ formamide, $0.9 \mathrm{M} \mathrm{NaCl}, 0.01 \mathrm{M}$ sodium dodecyl sulfate, and $20 \mathrm{mM}$ tris- $\mathrm{HCl}$ ) to remove extra probes.

\subsection{Image processing}

Sliced images in RGB (red-green-blue) mode are first converted to grayscale images to construct a histogram of pixels versus luminescence intensity by INSPECTOR (Matrox, Canada). Normally, the luminescence intensity distributed unevenly over the image from the phasecontrast images. A region of interest (ROI) was defined on the microtome-sliced image to include sufficient morphological detail for analysis, and also to avoid the inhomogeneity of the luminescent background [24]. In this study, the ROI was defined as a square of length based on volume-average size of flocs obtained from small-angle light scattering tests, termed as region $\mathrm{L}$, or on the gyration radius determined by SALS test, termed as region $\mathrm{S}$.

CLSM eliminated out-of-focus light at the lateral regions of the confocal plane and provided a homogeneously luminescent background. An ROI need not be selected on the CLSM sliced images. Otsu's method [25] could yield satisfactory thresholding among various histogram-based algorithms [24] and was chosen to estimate the floc porosity. The maximum convex perimeter method could probe the shapes and spatial distribution of the pores among the biomass granules, and was used herein to local structural profiling.

The area fractions of the white parts of the bilevel microtome-sliced images were taken as the values of "areal porosity" $\varepsilon_{2 \mathrm{D}}$. Once the boundaries of the bilevel image were available, the pores could be identified. The medium and volume-average pore sizes were determined. The boundary fractal dimension, $D_{\mathrm{B}}$, and Sierpinski carpet fractal dimension, $D_{\mathrm{SC}}$, of pore surface were estimated from the microtome-sliced images. For an object with rugged boundaries, the following relationship exists between the area and the perimeter [26],

$A_{\mathrm{p}} \propto p_{\mathrm{p}}^{2 / D_{\mathrm{B}}}$,

where $D_{\mathrm{B}}$ is the fractal dimension of the pore boundaries. Assuming that the cross-section of sludge floc can be described as a Sierpinski carpet, the Sierpinski carpet fractal dimension $D_{\mathrm{SC}}$ can be determined to quantify the "disappearing rate" of remaining area. The volume fractions of the white parts of the $3 \mathrm{D}$ reconstruction model were the values of "porosity" $\varepsilon_{3 \mathrm{D}}$. Two morphological parameters were used to describe the fractal behavior of the three-dimensional model constructed from the CLSM images. The compactness of a three-dimensional object is defined as $A^{3} / 36 \pi V^{2}$, where $A$ and $V$ are the surface area and the volume of the pores, respectively, that were determined in CLSM images. The compactness of each pore was calculated and the average of all pores was reported. The boxcounting fractal dimension $\left(D_{\mathrm{P}, 3}\right)$ of one pore is determined by [27]

$D_{\mathrm{P}, 3}=-l_{\text {equl }} \rightarrow 0 \lim \frac{\log N\left(l_{\text {equi }}\right)}{\log l_{\text {equi }}}$, 
where $N\left(l_{\text {equi }}\right)$ is the number of triangular patches covering the pores, and the characteristic side length is $l_{\text {equi. }}$. In practice, limit $l_{\text {equi }} \rightarrow 0$ cannot be approached. $D_{\mathrm{P}, 3}$ is estimated by measuring the dependence of $N$ on $l_{\text {equi }}$ when the characteristic length is larger than some cut-off length, which is the size in pixels of the original sliced image.

\section{Results and discussion}

\subsection{Microphotographs}

The phase contrast microphotographs of original and conditioned sludge flocs can be found elsewhere and are not shown here for brevity [24]. Cationic polyelectrolyte flocculation significantly increased floc sizes by polymer bridging. The flocs became compact as many original flocs stacked on each other. Freeze/thawed sludge flocs were also larger and more compact.

\subsection{Dewaterability}

The dewatering performance improved markedly as the sludge was conditioned. The filterability, $\chi$, was improved from $5.6 \times 10^{-6}$ to $21 \times 10^{-6}$ (flocculated) or $27 \times 10^{-6} \mathrm{~kg}^{2} \mathrm{~s}^{-2} \mathrm{~m}^{-4}$ (freeze/thawed). The zone settling velocity (ZSV) was enhanced by $1000-1500$ times after conditioning. Moreover, the final sediment heights were considerably reduced when physical or chemical conditioning was applied.

\subsection{Small-angle light scattering tests}

Table 1 summarizes the floc sizes from SALS tests. The results indicate that floc size increased substantially after flocculation or freezing and thawing.

The original flocs exhibited a $D_{\mathrm{S}}$ of 2.18 (Fig. 1). The scattering fractal dimension $D_{\mathrm{S}}$ of both flocculated sludge (1.94) and freeze/thawed sludge (1.95) decreased. Wu et al. [9] used SALS to examine structural changes of waste-activated sludge under cationic flocculation with the same flocculant. They reported that as the flocculant dose was increased, $D_{\mathrm{S}}$ first decreased and then increased above some critical dose. The fall in $D_{\mathrm{S}}$ was possibly due to the looseness of the aggregate structure after polymer conditioning.

\subsection{Free settling tests}

Based on Eq. (2), the flocculated sludge had a lower $D_{\mathrm{F}}$ (1.30) (Fig. 2b) than the original sludge (1.47) (Fig. 2a), while the freeze/thawed sludge had a higher value (1.55) (Fig. 2c). $D_{\mathrm{F}}$ could be considered to be a geometric parameter that describes the configuration of the entire floc [9]. The freeze/thawed sludge flocs should have been subjected to interfacial dehydration and exhibited a relatively compact structure, of which the porous configuration was compressed and collapsed [28]. Hence, a high $D_{\mathrm{F}}$ for freeze/thawed flocs was determined in the free-settling tests.

Table 1

The free-settling and SALS tests and the morphological parameters of two-dimensional image from waste-activated sludge flocs

\begin{tabular}{llll}
\hline & Original & Cationic flocculated & Freeze/thawed \\
\hline SLS and free-settling tests ${ }^{\mathrm{a}}$ & & & \\
$d_{\mathrm{f}}(0.5)(\mu \mathrm{m})$ & $87.4 \pm 26$ & $989 \pm 73$ & $752 \pm 74$ \\
$d_{\mathrm{f}}[4,3](\mu \mathrm{m})$ & $122 \pm 28$ & $986 \pm 52$ & $795 \pm 47$ \\
$R_{\mathrm{f}}(\mu \mathrm{m})$ & 32.3 & $\mathrm{NA}$ & $\mathrm{NA}$ \\
$R_{\mathrm{p}}(\mu \mathrm{m})$ & 0.57 & 0.48 & 0.49 \\
$D_{\mathrm{S}}(-)$ & $2.18 \pm 0.02$ & $1.94 \pm 0.05$ & $1.95 \pm 0.01$ \\
$D_{\mathrm{F}}(-)$ & 1.47 & 1.30 & 1.55 \\
& & & \\
$M_{\text {orphological parameters }}{ }^{\mathrm{b}}$ & & & $0.65 \pm 0.03$ \\
$\varepsilon_{2 \mathrm{D}}^{(\mathrm{S})}$ & $0.73 \pm 0.04$ & $0.68 \pm 0.03$ & $0.63 \pm 0.03$ \\
$\varepsilon_{2 \mathrm{D}}$ & $0.71 \pm 0.01$ & $0.71 \pm 0.02$ & $17.5 \pm 8.3$ \\
$d_{\mathrm{p}}[4,3]^{(\mathrm{L})}(\mu \mathrm{m})$ & $23.6 \pm 9.0$ & $59.3 \pm 23.2$ & $10.3 \pm 3.8$ \\
$d_{\mathrm{p}}[4,3]^{(\mathrm{S})}(\mu \mathrm{m})$ & $6.4 \pm 2.3$ & $11.4 \pm 6.2$ & $1.76 \pm 0.06$ \\
$D_{\mathrm{SC}}^{(\mathrm{LC})}$ & $1.72 \pm 0.08$ & $1.81 \pm 0.03$ & $1.73 \pm 0.03$ \\
$D_{\mathrm{SC}}^{(\mathrm{S})}$ & $1.74 \pm 0.03$ & $1.78 \pm 0.04$ & $1.35 \pm 0.01$ \\
$D_{\mathrm{B}}^{(\mathrm{L})}$ & $1.35 \pm 0.06$ & $1.48 \pm 0.08$ & $1.34 \pm 0.01$ \\
$D_{\mathrm{B}}^{(\mathrm{S})}$ & $1.38 \pm 0.01$ & $1.38 \pm 0.08$ & \\
\hline
\end{tabular}

\footnotetext{
${ }^{a} \mathrm{NA}$ : The Guinier regime does not appear on the $I$ vs. $Q$ plot so that $R_{\mathrm{f}}$ cannot be estimated.

${ }^{\mathrm{b}}$ The superscripts ${ }^{(\mathrm{L})}$ and ${ }^{(\mathrm{S})}$ denote the data are obtained from the images of magnification $100 \times(0.29 \mu \mathrm{m} /$ pixel $)$ and $400 \times(0.07 \mu \mathrm{m} /$ pixel $)$, respectively.
} 


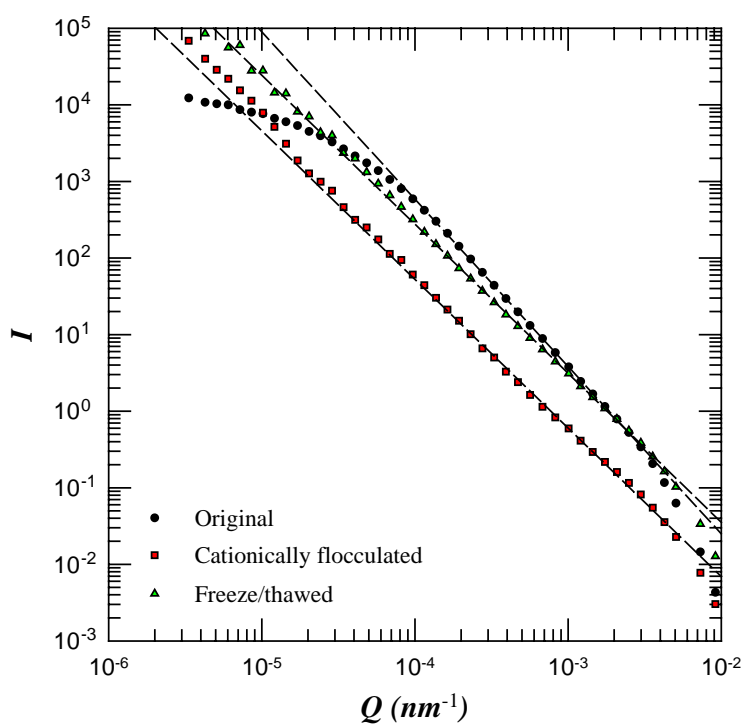

Fig. 1. $I$ vs. $Q$ plot of waste-activated sludges prior to and after conditioning.

\subsection{Microtome slicing}

Fig. 3 presents several examples of typical microtome slices. The ROIs to be analyzed were determined using the proposed image-processing algorithm. For samples with undetermined $R_{\mathrm{f}}$ (freeze/thawed and flocculated ones), the $R_{\mathrm{f}}$ of original sludge was used to determine the size of ROI. For original sludge flocs, the microphotograph with a magnification of $100 \times$ (Fig. 3a) displayed a cluster of mass with open structures. At a higher magnification of $400 \times$ (Fig. 3b), many "cores" (biomass granules) with concentrated biomass, as described by $\mathrm{Li}$ and Ganczarczyk [1], were noted. Pores distributed among the biomass granules were of sizes $20-40 \mu \mathrm{m}$. Smaller pores were "hidden" in the granules. The sizes of the large and small pores in the original sludge floc differed significantly. Some fiber-like matter, possibly the remains of filamentous bacteria, was scattered around biomass granules.

Cationically flocculated sludge flocs (nearly $1000 \mu \mathrm{m}$ ) were larger than the size of Fig. 3c. Some large pores are larger than $50 \mu \mathrm{m}$, possibly favoring settling. Some compact regions were also observed (for example, the lower-right side of Fig. 3d). Although the biomass is distributed less uniformly than that of the original sludge floc, the slice appeared to be basically similar to the original floc clusters in Fig. 3a. Magnifying the compact regions of the flocculated sludges, as in Fig. 3d, showed few pores of size $20 \mu \mathrm{m}$, and the biomass cores became much larger than those of original flocs

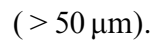
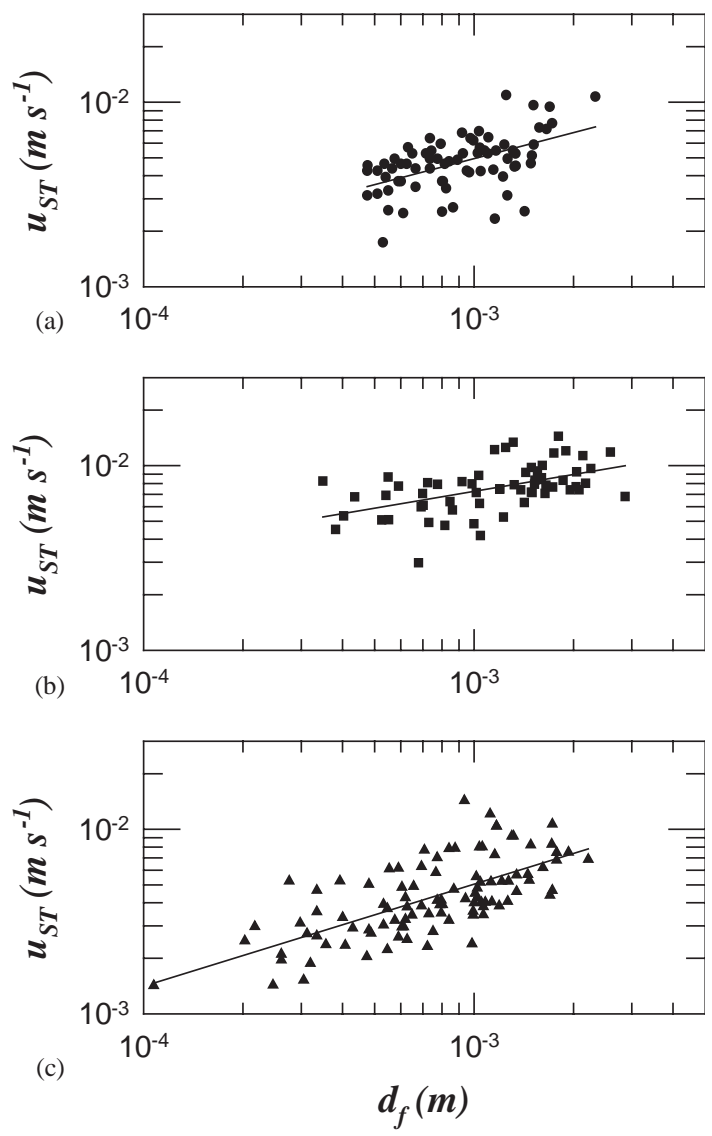

Fig. 2. Free-settling tests of the waste-activated sludges subjected to conditioning: (a) original; (b) cationically flocculated; (c) freeze/thawed.

Freeze/thawed sludge flocs displayed very compact morphology in both microphotographs (Figs. 3e and 3f). In Fig. 3f, most pores were smaller than $5 \mu \mathrm{m}$. Biomass granules of size $80 \mu \mathrm{m}$ had a much darker color than the original and flocculated sludge. Although the biomass concentration was not directly proportional to the luminescence intensity, more concentrated biomass of the freeze/thawed sludge flocs was still imaged.

Table 1 summarizes morphological parameters of the (two-dimensional) sliced images. Both the original and conditioned flocs had a two-level structure [9], evidenced by the different floc characteristics in regions $\mathrm{L}$ and $\mathrm{S}$. Except for the flocculated sludge flocs, $\varepsilon_{2 \mathrm{D}}^{(\mathrm{L})}$ was higher than $\varepsilon_{2 \mathrm{D}}^{(\mathrm{S})}$, indicating that the basic units in flocs have more compact structures than the entire matrices for original and freeze/thawed flocs. In region $\mathrm{L}$, the original sludge flocs had the highest $\varepsilon_{2 \mathrm{D}}^{(\mathrm{L})}(0.733$ in average), falling to 0.684 for flocculated sludge and 0.647 for freeze/thawed sludge flocs. The original sludge flocs had a volume-average pore size $\left(d_{\mathrm{p}}[4,3]\right)$ of $23.6 \mu \mathrm{m}$, measured as the estimated distance among the biomass 

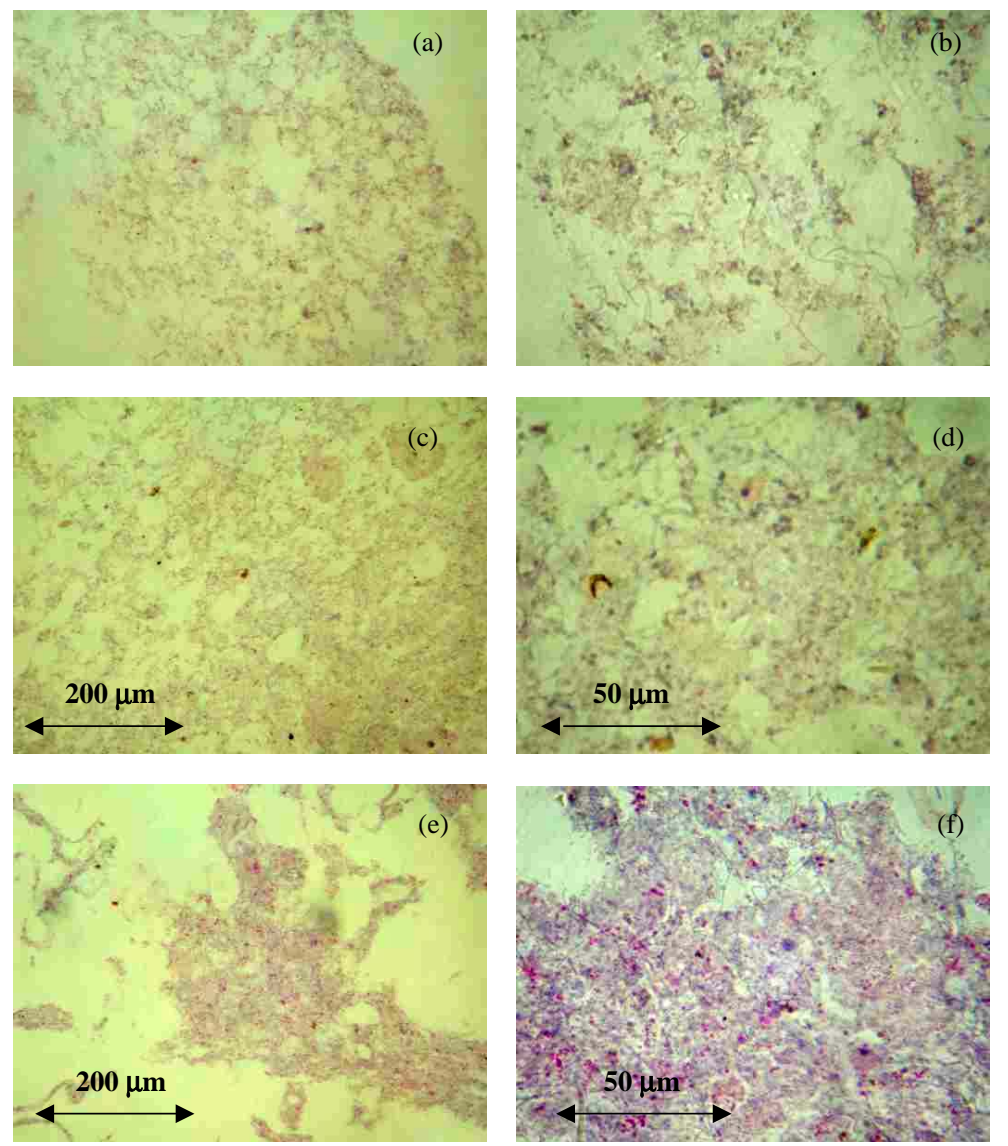

Fig. 3. The sliced images of original sludge: (a) Original floc, $100 \times$; (b) original floc, $400 \times$; (c) flocculated floc, $100 \times$; (d) flocculated floc, $400 \times$; (e) freeze/thawed floc, $100 \times$; (f) freeze/thawed floc, $400 \times$.

granules. The average pore size of sludge flocs increased to $59.3 \mu \mathrm{m}$ after flocculation. The correspondingly large standard deviation $(23.2 \mu \mathrm{m})$ indicated a highly nonuniform biomass distribution in the flocculated flocs. Some very large pores may have been formed because interstices among the original flocs were involved in the large matrices of flocculated flocs. The average pore size in the sludge flocs decreased to $17.5 \mu \mathrm{m}$ after freezing and thawing, implying that the biomass in the flocs was compressed during freezing, leading to a collapse in the global configuration. Meanwhile, the flocculated sludges had a higher $D_{\mathrm{SC}}^{(\mathrm{L})}$ and $D_{\mathrm{B}}^{(\mathrm{L})}$ than the original flocs, revealing a more "space-filling" pore structure with irregular and rugged pore boundaries. Freezing and thawing would have milder effect on the "roughness" of pore surface.

On the length scale of scattering aggregates (region S), the original and flocculated sludge flocs had higher $\varepsilon_{2 D}(\mathrm{D})$ than the freeze/thawed flocs, implying structural shrinkage in local regions after freezing. Cationic flocculation and freezing and thawing increased the average size of pores from 6.4 to $10-11 \mu \mathrm{m}$ in the scattering aggregates. The $D_{\mathrm{SC}}^{(\mathrm{S})}$ and $D_{\mathrm{B}}^{(\mathrm{S})}$ data revealed that, although the flocculation or freezing and thawing enlarged the fine pores, they had not considerably altered the pore structure.

\subsection{D floc model}

Fig. 4a displays a typical CLSM image. Using the Otsu's method, thresholding values of sliced images were determined. Fig. 4b shows the corresponding bilevel images of Fig. 4a. The 3D visualization and modeling software, Amira 3.0 (TGS Inc., USA), was used to reconstruct the thresholded sliced images as isosurfaces (polygonal surface models). The following procedure was employed: (1) The resolution of the image was adjusted to make the physical shape of the voxel to almost a cube. The Lanczos filter is used in the resampling. (2) The connectivity of neighboring pixels in all analyses is set to four for edge detection. The edges in the images are white lines in Fig. 4c. (3) The surface 


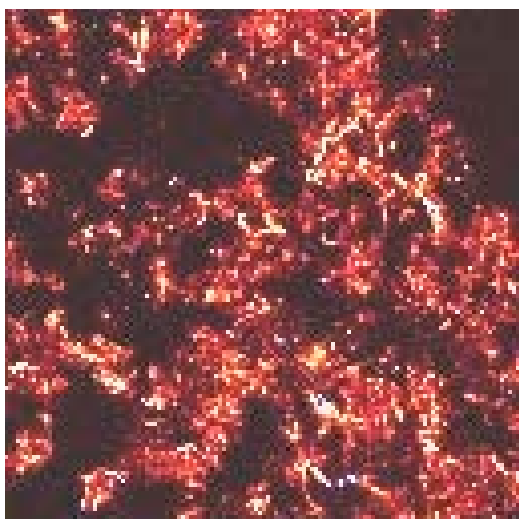

(a)

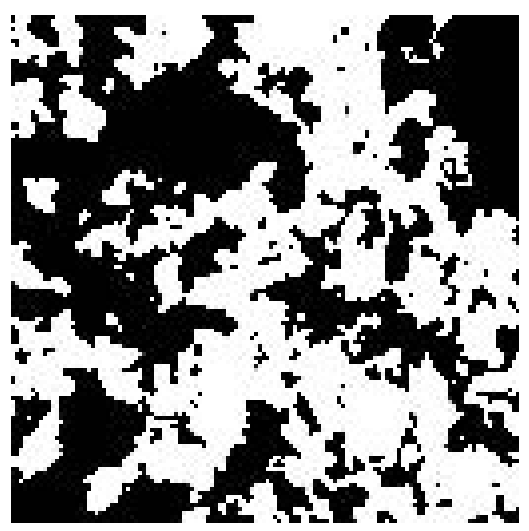

(b)

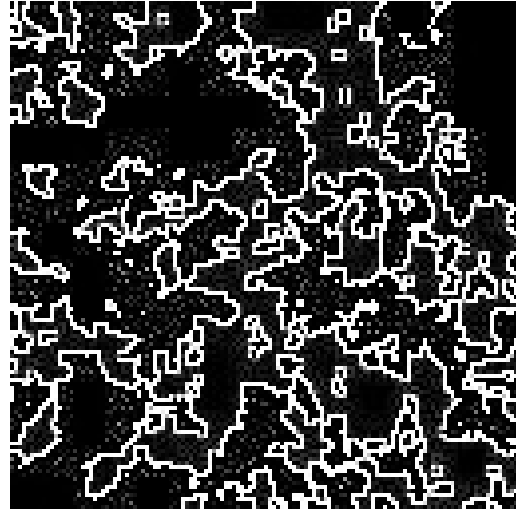

(c)

Fig. 4. A series of CLSM images at the interval of $17 \mu \mathrm{m}$. Image width $=178 \mu \mathrm{m}$.

construction algorithm adopted here is the marching cubes algorithm for triangulating surfaces.

Fig. 5 displays CLSM images, thresholded images and three-dimensional views of the biomass portion of the selected, original flocs. The images for conditioned flocs are not shown for brevity sake. Table 2 presents the geometric parameters derived from these models. The size represents length $(X)$, width $(Y)$ and height $(Z)$ of a cuboid model. This study used a $40 \times$ objective providing an analytical range of less than $300 \mu \mathrm{m}$. Fig. 5 exhibits the complex porous configurations of the flocs. Since clearly distinguishing the volumes "inside" and "outside" the floc on CLSM sliced images is difficult, the model generally includes a floc and the surrounded fluid, and thus the floc does not occupy the entire interior of the model.

All geometric parameters evaluated depended on the adopted resolution. Fig. 6 shows the volume porosity data estimated based on various voxel lengths. Since the detailed structure contributed little to the total void space, the porosities depends weakly on the image resolutions. Since the gyration radius $R_{\mathrm{p}}$ is around $0.5 \mu \mathrm{m}$, a length scale of $l_{\text {eaui }}=1 \mu \mathrm{m}$ was chosen to evaluate all geometric parameters.

The box-counting fractal dimension $D_{\mathrm{P}, 3}$ can be estimated from Fig. 7. The original sludge flocs had $D_{\mathrm{P}, 3}$ of about 2.57. Similar to the results for $2 \mathrm{D}$ analysis, cationic flocculated sludge flocs with more irregular surfaces had the highest $D_{\mathrm{P}, 3}(2.62-2.70)$, and freeze/ thawed sludge flocs had lower $D_{\mathrm{P}, 3}(2.45-2.50)$ than the original flocs.

Fig. 8 shows the surface area of all pores versus the voxel length. The surface area obtained with $l_{\text {equi }}=1 \mu \mathrm{m}$ was used to calculate the three-dimensional compactness. The original sludge flocs had a compactness between 30 and 50 (Table 2), whereas the compactness of flocculated sludge flocs was extremely high (400-
1200). Hence, the action of flocculation yielded internal pores of shape resembling a long "tube". In contrast, the compactness of the freeze/thawed flocs is slightly lower (19-33) than the original flocs, implying that the internal pore has a lower aspect ratio. This information together with the fractal dimension data, compared with the original flocs, revealed that the flocculation produced pores of larger aspect ratio (length/diameter) and with rough surface; while the freezing and thawing yielded internal pores of smaller aspect ratio and with smoother surface.

\subsection{Discussion}

Flocs of higher $D_{\mathrm{F}}$, such as freeze/thawed samples, have a lower $D_{\mathrm{P}, 3}, d_{\mathrm{p}}^{(\mathrm{L})}$, and compactness. However, a simple correlation between $D_{\mathrm{F}}$ and either $\varepsilon_{2 \mathrm{D}}^{(\mathrm{L})}$ or $\varepsilon_{3 \mathrm{D}}$ is hard to derive. Inconsistent with the generally accepted hypothesis, flocs with compact configurations (lower $\left.\varepsilon_{2 \mathrm{D}}^{(\mathrm{L})}\right)$ did not always have higher $D_{\mathrm{F}}$ values than the loose flocs. For example, flocculated and freeze/thawed sludge flocs had a lower porosity than original sludge flocs. The former had a lower $D_{\mathrm{F}}$, but the latter had higher $D_{\mathrm{F}}$. Apparently, neither $\varepsilon_{2 \mathrm{D}}^{(\mathrm{L})}$ nor $\varepsilon_{3 \mathrm{D}}$ singly governs the variation of $D_{\mathrm{F}}$. A floc with larger $d_{\mathrm{p}}^{(\mathrm{L})}$ and compactness exhibits lower $D_{\mathrm{F}}$ than another whose floc size is of the same order of magnitude. This finding is pertinent to comparisons between the flocculated sludge and freeze/ thawed sludge flocs. A floc could only have a few long and large pores, through which most of the advective water could flow. These pores could have a chance to be loosely allocated within the floc, reflecting to a low $D_{\mathrm{F}}$. The free-settling behavior was determined by internal porous configuration, rather than porosity $\left(\varepsilon_{2 \mathrm{D}}^{(\mathrm{L})}\right.$ or $\left.\varepsilon_{3 \mathrm{D}}\right)$ alone.

The $D_{\mathrm{S}}$ was neither correlated with the porosity of scattering aggregates $\left(\varepsilon_{2 \mathrm{D}}^{(\mathrm{S})}\right)$, nor related to $D_{\mathrm{B}}^{(\mathrm{S})}$. Although 

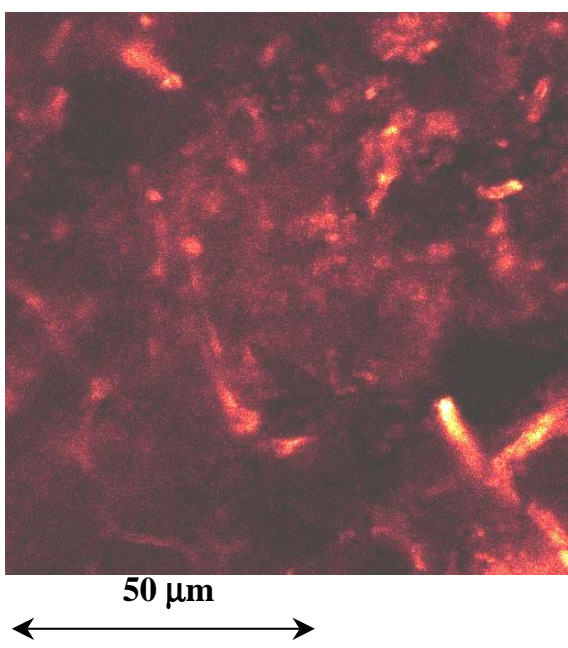

(a)

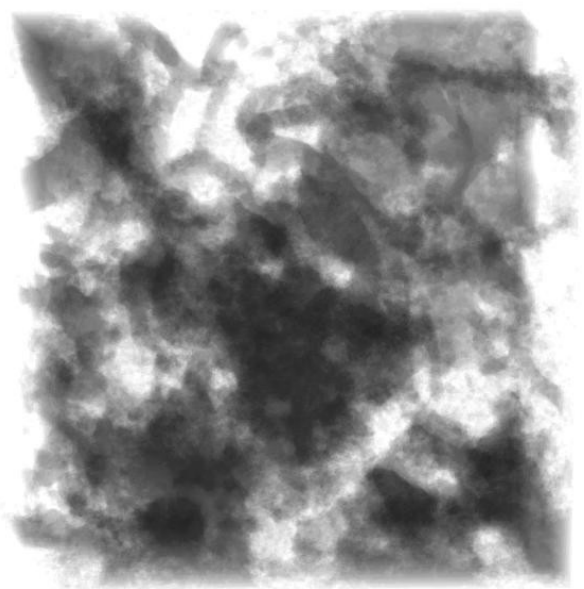

(c)

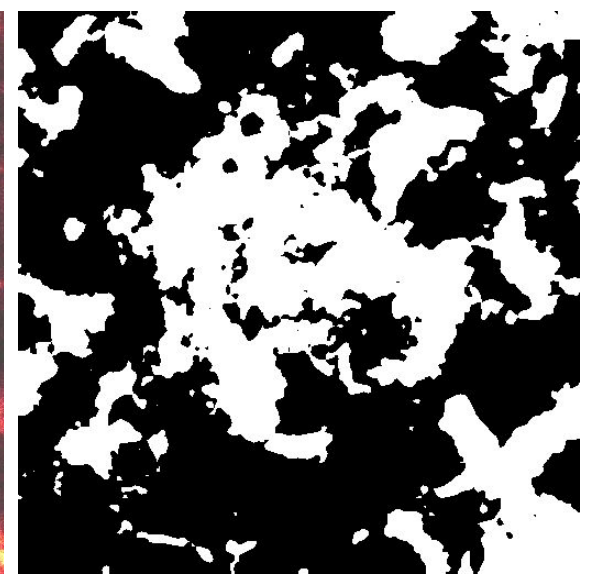

(b)

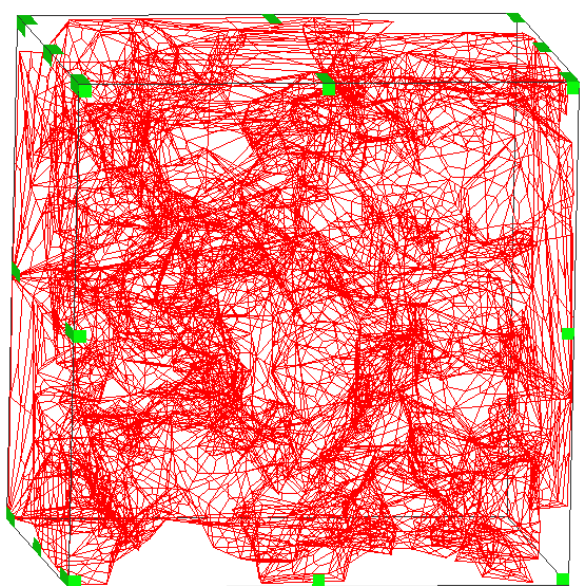

(d)

Fig. 5. Original floc B: (a) one typical CLSM sliced image; (b) bilevel image thresholded by Otsu's method; (c) three-dimensional view of floc biomass; (d) hexahedral volumetric grids of the pore.

the flocculated and freeze/thawed sludge flocs had lower porosity in scattering aggregates (lower $\left.\varepsilon_{2 \mathrm{D}}^{(\mathrm{S})}\right)$ than the original floc, the obtained values of $D_{\mathrm{S}}$ were still significantly lower than that of the original floc. Freeze/thawed flocs with a similar surface and flocculated flocs with rugged morphologies both had a lower $D_{\mathrm{S}}$ than the original flocs. Meanwhile, a larger average pore size $\left(d_{\mathrm{p}}[4,3]^{(\mathrm{S})}\right)$ was approximately correlated with a lower $D_{\mathrm{S}}$. The evidence presented herein could not comprehensively determine the relationship between $D_{\mathrm{S}}$ and other geometric parameters. Again, it is suggested that the flocs with larger local pores would exhibit a smaller $D_{\mathrm{S}}$. The intensity function $I$ in SALS tests might well be governed by the interactions between various parameters, including floc size, pore size and the number of scattering aggregates in the global structure of the floc. Conditioning, usually, considerably enlarges the floc, making the situation more complicated.

The detailed mechanisms on how conditioning alters the floc structure remain unclear. Nonetheless, the structural information presented herein provides a protocol on further development of floc model, and as a model system to analyze detailed intrafloc transport processes.

\section{Conclusions}

We examine how cationic polyelectrolyte flocculation or freezing and thawing affected the floc structure using free-settling tests, SALS test, microtome-slicing techniques, and CLSM experiments. Both flocculation and 
Table 2

The morphological parameters of the original, cationic flocculated, and freeze/thawed flocs

\begin{tabular}{|c|c|c|c|c|}
\hline No. & Size $(\mu \mathrm{m} \times \mu \mathrm{m} \times \mu \mathrm{m})$ & $\varepsilon_{3 \mathrm{D}}^{\mathrm{a}}$ & $D_{\mathrm{P}, 3}$ & Compactness \\
\hline \multicolumn{5}{|c|}{ Original flocs } \\
\hline A & $113 \times 113 \times 102$ & 0.63 & 2.56 & 50.8 \\
\hline B & $93.8 \times 93.8 \times 74.9$ & 0.64 & 2.57 & 37.7 \\
\hline $\mathrm{C}$ & $77.7 \times 77.7 \times 112$ & 0.64 & 2.58 & 31.8 \\
\hline \multicolumn{5}{|c|}{ Flocculated flocs } \\
\hline A & $238 \times 238 \times 198$ & 0.58 & 2.70 & 1,270 \\
\hline B & $188 \times 188 \times 132$ & 0.54 & 2.63 & 211 \\
\hline $\mathrm{C}$ & $178 \times 178 \times 134$ & 0.60 & 2.62 & 405 \\
\hline \multicolumn{5}{|c|}{ Freezelthawed flocs } \\
\hline A & $122 \times 122 \times 88.2$ & 0.62 & 2.46 & 25.1 \\
\hline B & $93.8 \times 93.8 \times 51.0$ & 0.61 & 2.46 & 19.3 \\
\hline $\mathrm{C}$ & $93.8 \times 93.8 \times 59.1$ & 0.60 & 2.51 & 33.6 \\
\hline
\end{tabular}

${ }^{a}$ The porosity indicated the volumetric percentage of the empty portion in the cuboids.

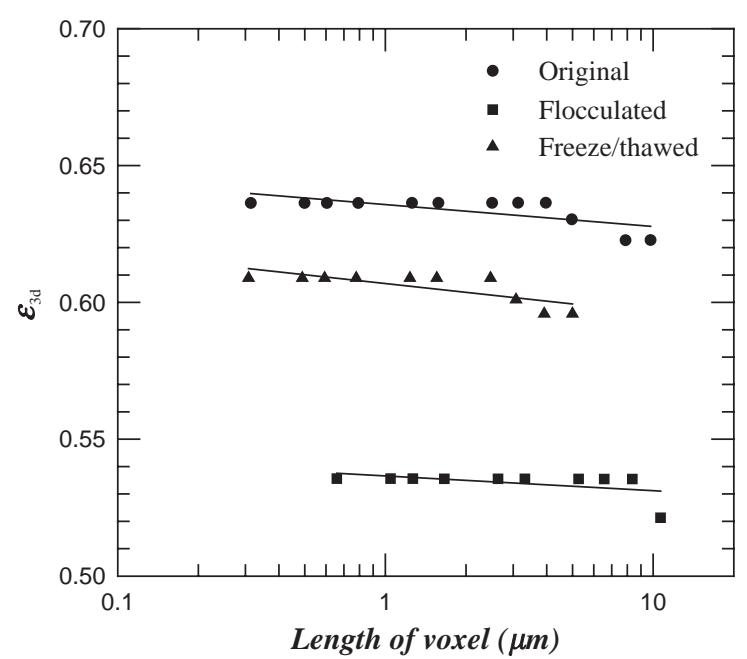

Fig. 6. The volume porosity data versus the equilibrium voxel size $l_{\text {equi. }}$

freeze/thawed treatment considerably enlarged the floc size and enhanced dewaterability. The former yielded a lower fractal dimension determined from free-settling test $\left(D_{\mathrm{F}}\right)$ and the latter produced a higher $D_{\mathrm{F}}$. Meanwhile, both treatments led to a lower fractal dimension measured from SALS test $\left(D_{\mathrm{S}}\right)$.

The microtome slices and CLSM images revealed a complex structure within sludge flocs. The geometric parameters for images probed at large scale differed from those at small scale, showing a two-level structure of sludge flocs. On large-scale probing, the flocculated floc was shown to have a compact structure with big

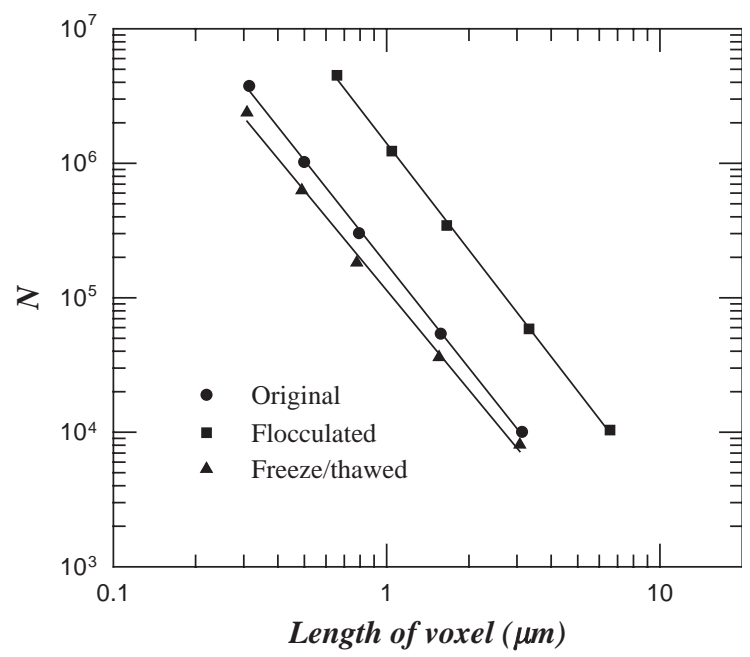

Fig. 7. The number of triangular patches on the constructed surface versus the equivalent voxel size $l_{\text {equi }}$.

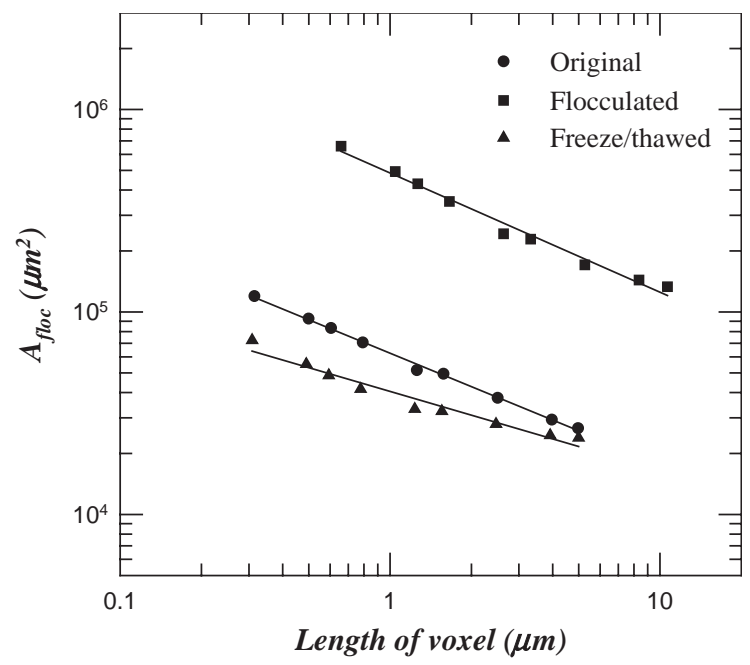

Fig. 8. The total surface area of pores identified versus the equilibrium voxel size $l_{\text {equi }}$.

internal pores of rugged boundary. The freeze/thawed flocs also produced compact structure, but the internal pores had smaller size and smoother boundary. On the length scale of scattering aggregates (region S), both cationic flocculation, and freezing and thawing increased the average size of internal pores from 6.4 to $10-11 \mu \mathrm{m}$.

Reconstructing the CLSM series images can obtain the three-dimensional modeling of the sludge flocs. Cationic polyelectrolyte flocculation links the original sludge flocs into a large networked structure with highly non-uniform mass distribution. The surface becomes highly rugged, and increases the pores' box-counting 
fractal dimension $\left(D_{\mathrm{P}, 3}\right)$. Meanwhile, these pores had a large aspect ratio, indicating that they resemble long, rugged tubes in floc. Freezing and thawing, on the other hand, compressed local structure during ice formation and propagation, hence producing pores of a small aspect ratio and smooth boundary.

Further comparison shows a correlation between the pore size and the measured mass fractal dimensions. Large internal pores could have a better chance to be loosely allocated within floc, thereby producing a lower mass fractal dimension $\left(D_{\mathrm{F}}\right.$ or $\left.D_{\mathrm{S}}\right)$.

\section{References}

[1] Li DH, Ganczarczyk JJ. Structure of activated sludge flocs. Biotechnol Bioeng 1990;35(1):57-65.

[2] Lee DJ, Chen GW, Liao YC, Hsieh CC. On the freesettling test for estimating waste activated sludge floc density. Water Res 1996;30(3):541-50.

[3] Logan BE. Environmental transport processes. New York: Wiley; 1999.

[4] Li XY, Logan BE. Collision frequencies of fractal aggregates with small particles by differential sedimentation. Environ Sci Technol 1997;31:1229-36.

[5] Li XY, Logan BE. Collision frequencies between fractal aggregates and small particles in a turbulently sheared fluid. Environ Sci Technol 1997;31:1237-42.

[6] Jorand F, Zartarian F, Thomas F, Block JC, Bottero JY, Villemin G, Urbain V, Manem J. Chemical and structural (2D) linkage between bacteria within activated sludge flocs. Water Res 1995;29(7):1639-47.

[7] Sanin FD, Vesilind PA. Synthetic sludge: a physical/ chemical model in understanding bioflocculation. Water Environ Res 1996;68(5):927-33.

[8] Gorczyca B, Ganczarczyk J. Structure and porosity of alum coagulation flocs. Water Qual Res J Canada 1999;34(4):653-66.

[9] Wu RM, Lee DJ, Waite TD, Guan J. Multilevel structure of sludge flocs. J Colloid Interfac Sci 2002;252(2):383-92.

[10] Zartarian F, Mustin C, Villemin G, Ait-Ettager T, Thill A, Bottero JY, Mallet JL, Snidaro D. Three-dimensional modeling of an activated sludge floc. Langmuir 1997; 13:35-42.

[11] Thill A, Wagner M, Bottero JY. Confocal scanning laser microscopy as a tool for the determination of $3 \mathrm{D}$ floc structure. J Colloid Interfac Sci 1999;220:465-7.

[12] Snidaro D, Zartarian F, Jorand F, Bottero JY, Block JC, Manem J. Characterization of activated sludge flocs structure. Water Sci Technol 1997;36(4):313-20.
[13] Thill A, Veerapaneni S, Simon B, Wiesner M, Bottero JY, Snidaro D. Determination Of structure of aggregates by confocal scanning laser microscopy. J Colloid Interfac Sci 1998;204:357-62.

[14] Chu CP, Lee DJ. Moisture distributions in sludges: effects of cationic polymer conditioning. J Environ Eng ASCE 1999;125(4):340-5.

[15] Jean DS, Lee DJ, Wu JCS. Separation of oil from oily sludge by freezing and thawing. Water Res 1999;33(7): 1756-9.

[16] Li X, Yuan Y. Settling velocities and permeabilities of microbial aggregates. Water Res 2002;36:3110-20.

[17] Tang P, Raper JA. Modelling the settling behaviour of fractal aggregates - a review. Powder Technol 2002;123: 114-25.

[18] Bushell GC, Yan YD, Woodfield D, Raper JA, Amal R. On techniques for the measurement of the mass fractal dimension of aggregates. Adv Colloid Interfac Sci 2002;95:1-50.

[19] Lee DJ, Hsu YH. Fluid flow in capillary suction apparatus. Ind Eng Chem Res 1992;31:2379-84.

[20] Vesilind PA. Capillary suction time as a fundamental measure of sludge dewaterability. J Water Pollut Control Fed 1998;60(2):215-20.

[21] Chui HK, Fang HHP. Histological analysis of microstructure of UASB granules. J Environ Eng ASCE 1994;120(5):1322-6.

[22] Carson FL. Histotechnology: a self-instructional text. Chicago, USA: ASCP (American Society of Clinical Pathologists) Press; 1990. p. 43-68. [Chapter 3].

[23] Sorensen AH, Torsvik VL, Torsvik T, Poulsen LK, Ahring BK. Whole-cell hybridization of methanosarcina cells with two new oligonucleotide probes. Appl Environ Microbiol 1997;63(8):3043-50.

[24] Chu CP. Effects of conditioning, digestion on sludge floc structure. Doctoral dissertation, Department of Chemical Engineering. National Taiwan University, Taipei, Taiwan, 2003.

[25] Otsu N. A threshold selection method from gray-level histogram. IEEE Trans. Syst Man Cybern 1979;9(1):62-6.

[26] Zahid WM, Ganczarczyk JJ. Fractal properties of the RBC biofilm structure. Water Sci Technol 1994;29 (10-11):271-9.

[27] Baveye P, Boast CW, Ogawa S, Parlange J-Y, Steenhuis T. Influence of image resolution and thresholding on the apparent mass fractal characteristics of preferential flow patterns in field soils. Water Resour Res 1998;34(11):278396.

[28] Lee DJ, Hsu YH. Fast freeze/thaw process on excess activated sludges: floc structure and sludge dewaterability. Environ Sci Technol 1994;28:1444-9. 